\title{
Stabilizing Interconnection Characterization for Multi-Agent Systems with Dissipative Properties
}

\author{
Sandra Hirche* Shinji Hara** \\ * Institute of Automatic Control Engineering, Technische Universität \\ München, D-80290 Munich, GERMANY (e-mail: s.hirche@ieee.org). \\ ** Department of Information Physics and Computing, The University \\ of Tokyo, Tokyo 113-8656, JAPAN \\ (e-mail: shinji_hara@ipc.i.u-tokyo.ac.jp)
}

\begin{abstract}
This paper addresses the problem of stabilization and output-synchronization for a network of interconnected nonlinear agents, where each agent is assumed to be dissipative with respect to a specified quadratic supply rate which may differ among the agents. Main results concern the characterization and design of the information exchange structure for stabilization. Applying a linear protocol here, the associated interconnection matrix is characterized based on LMI's, as well as on spectral properties. Additionally a synthesis based on LMI's under structural constraints is proposed. The results are validated in simulations.
\end{abstract}

\section{INTRODUCTION}

The strong recent interest in group synchronization/coordination problems arises from a large number of different phenomena in biological systems such as schooling behavior of animals, behavior of crowds, synchronization of neural oscillators in the brain, as well as from control design problems, such as load balancing in large scale communication networks and the coordination of multiple robots or vehicles, see e.g. Fax and Murray [2004], OlfatiSaber et al. [2007], Lee and Spong [2006], Ren and Beard [2004] and references therein. In such a problem setting multiple autonomously acting agents are considered. The goal is to achieve a global behavior of the group in the whole. Each agent, however, acts based only on the local information acquired by sensing and/or by communication with neighbour agents. The general interest is in the analysis and design of the interconnections between the multiple agents and their local control to achieve a global control goal. Many interesting results have been achieved by applying graph theory to study the necessary interconnection structure, e.g. in Fax and Murray [2004], OlfatiSaber et al. [2007]. In most of these works the behavior of each agent is assumed to follow a simple kinematic model of the form $\dot{x}=u$. Only few works consider more realistic dynamics of the agents. For example, flocking - the synchronization of velocity - of robotic agents with inertia is studied e.g. in Lee and Spong [2006], the formation of vehicles in Fax and Murray [2004], Ren and Beard [2004].

In this paper, the agents are considered to have possibly different dynamics. All of them are assumed to be dissipative with respect to a quadratic supply rate which may differ among the agents. For the derivation of stability and output-synchronization conditions no explicit system model knowledge and no explicit construction of a Lyapunov function is required, the knowledge of the supply rate is sufficient. In this sense, it differs from the approaches e.g. in Fax and Murray [2004], Lee and Spong [2006], Ren and Beard [2004] where specific model knowledge is used. Related to this work is Arcak [2007], Ihle et al. [2007], and Chopra and Spong [2006], where passivity - a special case of dissipativity - is applied as analysis and synthesis tool. Main contribution of this paper is the characterization of the information structure under the assumption of a linear protocol to achieve stability and output synchronization using LMI and spectral conditions. Furthermore, we give an LMI to design the interconnection matrix under structural constraints. The results are validated in a numerical simulation.

The remainder of this paper is organized as follows: After presenting the problem setting in Section 2, the main result in terms of an LMI analysis and synthesis approach is given in Section 3. In Section 4 spectral conditions are derived for a common bound on the supply rate, in Section 5 simulations to validate the results are provided.

\section{PROBLEM SETTING}

We consider a set of $N$ interconnected locally controlled agents. Each agent $i$ has the dynamics

$$
\begin{aligned}
\dot{x}_{i} & =f_{i}\left(x_{i}\right)+g_{i}\left(x_{i}\right) u_{i} \\
y_{i} & =h_{i}\left(x_{i}\right),
\end{aligned}
$$

with the input $u_{i} \in \mathcal{U}_{i} \subset \mathbb{R}^{m}$, the state $x_{i} \in \mathcal{X}_{i} \subset \mathbb{R}^{n}$, the output $y_{i} \in \mathcal{Y}_{i} \subset \mathbb{R}^{p}$. The function $f_{i}(\cdot) \in \mathbb{R}^{n}$ is assumed to be locally Lipschitz, $g_{i}(\cdot) \in \mathbb{R}^{n \times m}, h_{i}(\cdot) \in$ $\mathbb{R}^{p}$ continuous and for convenience $f_{i}(0)=0, h_{i}(0)=0$. The system is assumed to be reachable and zero state detectable. Throughout the paper we assume each agent $i$ is $\left(Q_{i}, S_{i}, R_{i}\right)$-dissipative. 


\section{$2.1(Q, S, R)$-dissipative Agents}

In this subsection mainly known results on dissipative systems are repeated for convenience. Therefore we consider the system (1) with the index $i$ dropped.

Define a supply rate $w: \mathbb{R}^{m} \times \mathbb{R}^{p} \rightarrow \mathbb{R}$ associated with the system which is locally integrable $\int_{t_{0}}^{t_{1}} w(u(t), y(t)) d t<\infty$ for all $t_{1} \geq t_{0}$, and $w(0,0)=0$.

Definition 1. (Sepulchre et al. [1997]). The system (1) is dissipative with respect to the supply rate $w(u, y)$, if there exists a positive definite function $V(x)$ called storage function, such that for all $x \in \mathcal{X}$

$$
V(x(T))-V(x(0)) \leq \int_{0}^{T} w(u(t), y(t)) d t
$$

for all inputs $u \in \mathcal{U}$ and all finite $T \geq 0$. If $V$ is differentiable then condition (2) can be replaced by $\dot{V}(x(t)) \leq w(u(t), y(t))$.

In this paper a quadratic supply rate is considered leading to the notion of a $(Q, S, R)$-dissipative system.

Definition 2. (Willems [1972a]). A system is $(Q, S, R)$ dissipative if it is dissipative with respect to the quadratic supply rate ${ }^{1}$

$$
w(u, y)=y^{T} Q y+2 y^{T} S u+u^{T} R u,
$$

with $R \in \mathbb{R}^{m \times m}, S \in \mathbb{R}^{p \times m}, Q \in \mathbb{R}^{p \times p}$, constant matrices and $Q=Q^{T}, R=R^{T}$ symmetric.

The class of $(Q, S, R)$-dissipative systems includes for example passive systems, sector bounded systems. For a comprehensive treatment of dissipative systems refer to Sepulchre et al. [1997], Willems [1972a], Willems [1972b]. Another important result concerning the stablity of $(Q, S, R)$ dissipative systems is presented in the following.

Proposition 1. (Hill and Moylan [1976]). The system (1) is $(Q, S, R)$-dissipative if and only if there exists a positive definite $\mathcal{C}^{1}$ function $V(\cdot)$, a real function $l(\cdot)$ and a matrix $W$ such that

$$
\begin{aligned}
L_{f} V=\left(\frac{\partial V}{\partial x}\right)^{T} f(x) & =h^{T}(x) Q h(x)-l^{T}(x) l(x) \\
L_{g} V=\left(\frac{\partial V}{\partial x}\right)^{T} g(x) & =2 h^{T}(x) S-2 l^{T}(x) W \\
R & =W^{T} W .
\end{aligned}
$$

From now on we consider each agent $i=1, \cdots, N$ to be $\left(Q_{i}, S_{i}, R_{i}\right)$-dissipative with a radially unbounded $\mathcal{C}^{1}$ positive definite storage function $V_{i}$. The agents are assumed to be dissipative with respect to possibly different supply rates $w_{i}\left(u_{i}, y_{i}\right)$ for the results of Section 3 and to have a common bound $w\left(u_{i}, y_{i}\right)$ on their supply rates in Section 4. Observe that if an agent $i$ is dissipative with respect to a supply rate $w_{i}\left(u_{i}, y_{i}\right)$ then it is also dissipative to any $w\left(u_{i}, y_{i}\right)$ if $w\left(u_{i}, y_{i}\right) \leq w_{i}\left(u_{i}, y_{i}\right)$ holds for for all $u_{i} \in \mathcal{U}_{i}, y_{i} \in \mathcal{Y}_{i}$ and initial states. If all agents $i=1, \cdots, N$ with possibly different supply rates have such a bound, we will refer to that as the common bound of supply rate.

For the simplicity of notation we consider each agent to be a single-input-single-output ${ }^{2}$ (SISO) system, i.e.

\footnotetext{
1 The time argument is dropped for convenient notation.

2 The results apply straightforwardly to the multi-input-multioutput case by appropriate change of notation to Kronecker algebra.
}

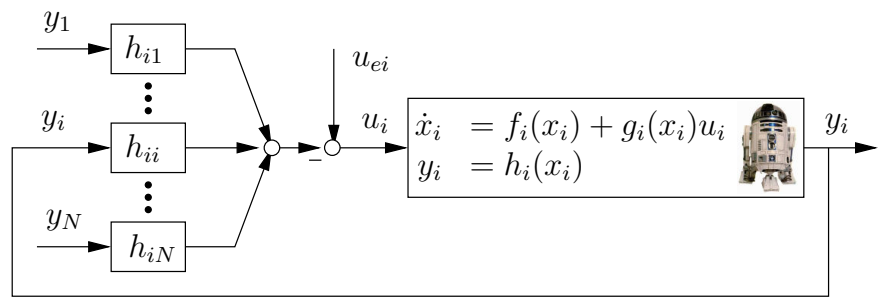

Fig. 1. Individual agent's control based on weighted outputs of other agents and local output feedback.

$m=p=1$. Define the diagonal matrices $Q=\operatorname{diag}\left\{Q_{i}\right\}$, $S=\operatorname{diag}\left\{S_{i}\right\}$, and $R=\operatorname{diag}\left\{R_{i}\right\}$ all $\in \mathbb{R}^{N \times N}$. As the system structure (1) does not contain any feedthrough term its follows that $R \geq 0$, and further we assume stable subsystems resulting in $Q \leq 0$.

\subsection{Interconnection of $N$ Agents}

A linear protocol is considered in this work; the interconnection is described by

$$
u_{i}=u_{e i}-\sum_{j=1}^{N} h_{i j} y_{j}, \quad i=1, \cdots, N
$$

where $u_{i}$ is the input to subsystem $i, y_{i}$ is its output, $u_{e i}$ is an external input, and the $h_{i j}$ are constant scalar gains. Stacking all outputs $y_{i}$ into a single vector $y=\operatorname{col}\left(y_{1}, \cdots, y_{N}\right)$, similarily for $u$ and $u_{e}$, the interconnection can be compactly rewritten as

$$
u=u_{e}-H y,
$$

with $H=\left\{h_{i j}\right\}$. Note that the diagonal elements $h_{i i}$ represent local feedback gains, the off-diagonal elements $h_{i j}$, $i \neq j$ represent the coupling gains. If the coupling gain $h_{i j}=0$, then there is no connection from subsystem $j$ to subsystem $i$.

In the remainder of the paper - if not further specified we refer to a general interconnection matrix $H$ without imposing any constraint. In some cases we make the following assumption

A1 The graph describing the agents' interconnections is strongly connected, and $H$ is the weighted graph Laplacian as defined in (A.1).

As a result of this assumption $H$ then has a simple eigenvalue at zero, the one-vector $\mathbf{1}^{T}=[1, \cdots, 1]$ is the associated righthand eigenvector, i.e. $H \mathbf{1}=0$. Further details on the graph-theoretic background refer to Appendix A.

The goal in the following is to characterize the interconnection matrix $H$ for which stability/output-synchronization is achieved. While asymptotic stability implies the convergence of the states to the origin, output-synchronization requires the difference of the outputs of any pair of agents to converge to zero as formally stated in the following.

Definition 3. (Chopra and Spong [2006]). Consider a system of $N$ interconnected agents as above. The agents are said to output-synchronize if $\lim _{t \rightarrow \infty}\left|y_{i}(t)-y_{j}(t)\right|=0$ for all $i, j=1, \cdots N$. 


\section{LMI CHARACTERIZATION FOR THE GENERAL CASE OF MULTIPLE SUPPLY RATES}

In this section we will derive our main result concerning the LMI characterization and also synthesis of the interconnection matrix $H$ for the network of agents with different supply rates to be stable/output-synchronizing.

\subsection{Interconnection Analysis}

Theorem 1. If there exists a diagonal matrix $D>0$ such that the matrix

$$
\hat{Q}=-H^{T} D R H+D S H+H^{T} S^{T} D-D Q
$$

is positive definite, i.e. $\hat{Q}>0$, then the network of $N$ interconnected $\left(Q_{i}, S_{i}, R_{i}\right)$-dissipative agents (1), (5) is asymptotically stable. If $\hat{Q} \geq 0$ is positive semidefinite while assumption A1 is satisfied then the agents outputsynchronize in the sense of Definition 3.

Proof. Let the Lyapunov function for agent $i$ denoted by $V_{i}\left(x_{i}\right)$. The overall Lyapunov function $V$ is choosen to be the weighted sum over all $V_{i}$, hence $V(x)=\sum_{i=1}^{N} d_{i} V_{i}\left(x_{i}\right)$ with $d_{i}>0$ and $x$ denoting the overall state vector containing all $x_{i}$. Using (4) and (6) with $u_{e} \equiv 0$ and setting $D=\operatorname{diag}\left\{d_{i}\right\}$ gives

$$
\begin{aligned}
\dot{V} & =\sum_{i=1}^{N} d_{i} \dot{V}_{i}\left(x_{i}\right) \\
& =u^{T} D R u+2 y^{T} D S u+y^{T} D Q y-\|\sqrt{D}(l(x)+W u)\|_{2}^{2} \\
& =-y^{T} \hat{Q} y-\|\sqrt{D}(l(x)-W H y)\|_{2}^{2} \\
& =\left[l^{T}(x) h^{T}(x)\right]\left[\begin{array}{cc}
-D & W D H \\
H^{T} D W & D Q-2 D S H
\end{array}\right]\left[\begin{array}{l}
l(x) \\
h(x)
\end{array}\right] \\
& =z^{T}(x) \Xi z(x)
\end{aligned}
$$

where $l(x)=\operatorname{col}\left(l_{1}\left(x_{1}\right), \cdots, l_{N}\left(x_{N}\right)\right)$ is the stacked vector consisting of the $l_{i}\left(x_{i}\right)$ and $W=\operatorname{diag}\left\{W_{i}\right\}$ is a diagonal matrix. Obviously, if $\hat{Q}>0$ then $\dot{V}<0$ for all $y \neq 0$. Zero state detectability ensures that $x \rightarrow 0$ as $t \rightarrow \infty$, i.e. the system is asymptotically stable. If $\hat{Q} \geq 0$ then $\dot{V} \leq 0$. Specifically, $\dot{V}(x) \equiv 0$ for all $x \in \Omega_{\Xi}$ with $\Omega_{\Xi}=\left\{x \in \mathbb{R}^{N n} \mid \Xi z(x)=0\right\}$. Consider now that assumption A1 holds. Then there are two cases: a) If at least one of the diagonal entries of $Q$ is negative, i.e. $\exists i, i \in\{1, \cdots, N\}$ so that $Q_{i}<0$ then $z(x)=\mathbf{0}$, hence $h(x)=\mathbf{0}$. Output synchronization is achieved with $\left|y_{i}\right|=\left|y_{j}\right|=0$. b) If all diagonal entries of $\mathrm{Q}$ are zero, the nullspace of $\Xi$ is one-dimensional and spanned by the vector $z^{T}(x)=\left[l^{T}(x) h^{T}(x)\right]=\left[\begin{array}{ll}\mathbf{0}^{T} & \mathbf{1}^{T}\end{array}\right]$. The set $\Omega_{\Xi}$ is characterized by the corresponding inverse function $z^{-1}(\cdot)$. The system converges to the largest invariant set within $\Omega_{\Xi}$ (LaSalle invariance principle) and outputsynchronization is achieved.

Remark 1. The above theorem is an extension to the result presented in Moylan and Hill [1978]. Here we use a weighted overall Lyapunov function and additionally consider the case of output-sychronization. Other related works are Arcak [2007], Ihle et al. [2007], and Chopra and Spong [2006] where passive agents are investigated.

Remark 2. Observe that the set of matrices $H$, i.e. the information structure, satisfying (7) in Theorem 1 is given by a matrix ellipsoidal set

$$
\left(H-H_{0}\right)^{T} R_{D}\left(H-H_{0}\right) \leq \bar{R}
$$

with the radius $\bar{R}=H_{0}^{T} R_{D} H_{0}-Q_{D}, Q_{D}=Q D$, and $H_{0}=R^{-1} S, R_{D}=R D$. The matrix ellipsoid is a compact convex set. It is non-empty, i.e. a solution $H$ satisfying (7) exist, if and only if the radius $\bar{R} \geq 0$ is positive semidefinite, see e.g. Peaucelle et al. [2002], being equivalent with requiring $S^{2}-R Q \geq 0$. This is always true as $Q \leq 0$ and $R \geq 0$. Consequently, a non-trivial interconnection matrix $\bar{H}$ satisfying Theorem 1 does always exist.

The above result can now be used to analyze the interconnection for different types of dissipativity of the individual agents. Exemplarily, the passivity case is presented here.

Corollary 1. The network of $N$ interconnected passive agents is asymptotically stable if the negative interconnection matrix $-H$ is strictly diagonally stable, i.e. there exists a positive definite diagonal matrix $D>0$ such that

$$
-D H-H^{T} D<0 \text {. }
$$

If $-D H-H^{T} D \leq 0$ and assumption A1 holds, then the agents output-synchronize.

Proof. Straightforward by applying Theorem 1 to passive agents, i.e. setting $Q=0, S=\frac{1}{2} I$, and $R=0$.

Remark 3. The case of passive agents is also considered by Chopra and Spong [2006] where balancedness of the graph, i.e. $H+H^{T} \geq 0$ is required for outputsynchronization. This is equivalent to requiring diagonal stability with $D=I$ in Corollary 1 . The following example shows the benefit of using the weighted Lyapunov approach which then leads to the above requirement of diagonal stability.

Example 1. Consider

$$
H=\left[\begin{array}{cc}
1 & -1 \\
-10 & 10
\end{array}\right] ; \quad D=\left[\begin{array}{cc}
10 & 0 \\
0 & 1
\end{array}\right]
$$

and observe that the interconnection matrix $H$ satisfies assumption A1. $H$ is not balanced, but satisfies $-D H-H^{T} D \leq 0$, i.e. $-H$ is diagonally stable and the agents output-synchronize.

For the stability/output-synchronization analysis problem the condition in Theorem 1 straightforwardly translates into an LMI feasibility problem. Consider the agents supply rate in $Q, S, R$ and an interconnection matrix $H$ given. Observe that the matrix $\hat{Q}(7)$ is linear in $D$. If the LMI $\hat{Q}>0$ admits a positive definite diagonal solution $D$ then the interconnection matrix $H$ asymptotically stabilizes the system. If the interconnection matrix $H$ satisfies assumption A1, and further the LMI $\hat{Q} \geq 0$ is feasible, then the interconnected system achieves outputsynchronization.

\subsection{Interconnection Synthesis}

In the interconnection synthesis problem we jointly search for a positive diagonal solution $D$ and the interconnection matrix $H$ for given $Q, S, R$ containing the parameters of the possibly different agent supply rates.

Theorem 2. Consider a network of $N$ interconnected $\left(Q_{i}, S_{i}, R_{i}\right)$-dissipative agents. The interconnection matrix $H$ asymptotically stabilizes the overall system if there exist a solution to the LMI

$$
Y>0 \quad\left[\begin{array}{cc}
S X+X^{T} S-Q Y & X^{T} R^{\frac{1}{2}} \\
R^{\frac{1}{2}} X & Y
\end{array}\right]>0
$$


with $Y$ diagonal. If $H$ satisfies the second LMI non-strict $(\geq)$ under the constraint A1 then the agents outputsynchronize in the sense of Definition 3. The interconnection matrix is given by $H=X Y^{-1}$.

Proof. Most arguments for the proof are similar to the one for Theorem 1. Here we only show that the above LMI condition implies the positive definiteness/ semi-definiteness of (7). Under the change of variables $X=H D^{-1}, Y=D^{-1}$ and using the Schur complement the statement is equivalent to the existence of a diagonal $D>0$ such that $-D^{-1} H^{T} D R H D^{-1}+S H D^{-1}+$ $D^{-1} H^{T} S-Q D^{-1} \geq 0$ is satisfied, analogously for strict inequality. By left- and right-multiplying $D$ this matrix inequality shows to be equivalent to (7) in Theorem 1.

Remark 4. Note that as $Y$ is diagonal, the structure of the interconnection matrix $H=X Y^{-1}$ can be pre-defined by partially or fully setting the structure for the solution $X$. If for example a certain sparse interconnection structure is preferred certain interconnections between agents can be inhibited by adding the equality constraint

$$
x_{i j}=0 \text { for }(i, j) \notin \mathcal{E}^{*}
$$

to the LMI (9) where the set $\mathcal{E}^{*}$ of all admissible edges represents the admissible graph structure. With similar arguments the requirements for $H$ imposed by assumption A1 in the second part of the Theorem 2 can be realized by constraining the solution space for $X$. Current research is on how advanced performance criteria can be implemented in a similar fashion as for the graph Laplacian in Boyd [2006].

\section{SPECTRAL CHARACTERIZATION FOR COMMON BOUND OF SUPPLY RATE}

Spectral graph theory is an interesting tool and has been applied to characterize information structures for outputsynchronization in earlier works, e.g. in Fax and Murray [2004]. In this section we investigate to which extent it is possible to characterize the stabilizing information structure by the spectrum $\sigma(H)$ of the interconnection matrix $H$ under the given system assumptions. Therefore we now consider $\left(Q_{i}, S_{i}, R_{i}\right)$-dissipative agents with the common bound $w\left(u_{i}, y_{i}\right)$ on the supply rate which is characterized by $(q, s, r)$, hence $Q=q I, S=s I$, and $R=r I$. This assumption simplifies the stability/outputsynchronization condition (7) from Theorem 1 to

$$
\hat{Q}_{q s r}=-r H^{T} D H+s\left(D H+H^{T} D\right)-q D>0,
$$

for some diagonal $D>0$ and $\hat{Q}_{q s r} \geq 0$. Note that the existence of a positive definite symmetric solution $D$ in (10) is equivalent to the spectrum of $H$ belonging to an algebraic domain $\mathbb{D}(p)$ as stated in Lemma 2. Accordingly, if (10) admits a diagonal solution $D$ then the spectrum of the associated $H$ also belongs a specific algebraic domain which is described in the following. The opposite, however, is not true.

In order to demonstrate that consider the set $\mathcal{M}_{\sigma(M)}$ of all real matrices sharing the same spectrum $\sigma(M)$. Then for any two matrices $A, B \in \mathcal{M}_{\sigma(M)}$ there exists a nonsingular real matrix $T$ such that $A=T B T^{-1}$. Assume that the matrix $H$ satisfies the matrix inequality (10) for some diagonal $D>0$. Inserting $H=T \tilde{H} T^{-1}$, hence

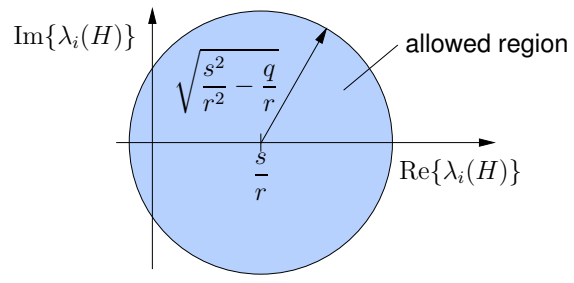

Fig. 2. Spectral location of the eigenvalues of the interconnection matrix $H$ for output-synchronization of network of $(q, s, r)$-dissipative agents.

$\tilde{H} \in \mathcal{M}_{\sigma(H)}$, and left-, right-multiplying (10) with $T^{T}$, $T$ respectively gives the equivalent expression

$$
-r \tilde{H}^{T} \tilde{D} \tilde{H}+s\left(\tilde{D} \tilde{H}+\tilde{H}^{T} \tilde{D}\right)-q \tilde{D}>0
$$

with $\tilde{D}=T^{T} D T$. Clearly, $D>0$ implies $\tilde{D}>0$, accordingly for $\hat{Q}_{q s r} \geq 0$. It is obvious, however, that not every non-singular transformation matrix $T$ will render $\tilde{D}$ diagonal which is required for Theorem 1 to hold. The following lemma describes the appropriate class of transformations.

Lemma 1. Let $D>0$ be a diagonal matrix and consider the set of matrices $\mathcal{T}(D)=\left\{T \mid T=D^{-\frac{1}{2}} U \Theta\right\}$ with some orthogonal matrix $U^{T}=U^{-1}$ and some diagonal matrix $\Theta=\operatorname{diag}\left\{\theta_{i}\right\}, \theta_{i} \neq 0$ for all $i$. If $T \in \mathcal{T}(D)$ then $\tilde{D}=T^{T} D T=\Theta^{2}$ is diagonal.

As a result, if some matrix $H$ satisfies (10) for some diagonal $D>0$ then not all co-sprectral matrices, i.e. from the set $\mathcal{M}_{\sigma(H)}$, also satisfy that. Hence, the spectrum of the interconnection matrix $H$ alone does not represent a sufficient condition for stability. If a matrix $H$ admitting the diagonal solution $D>0$ to (10) is known, then the set of co-spectral matrices also admitting a diagonal solution is specified by $\tilde{H}=T^{-1} H T$ with $T \in \mathcal{T}(D)$, $\tilde{D}=T^{T} D T>0$.

So far the class of matrices for a specific spectrum were analyzed, now we are interested in the bounds of the spectrum $\sigma(H)$ such that a solution to (10) exists. Therefore consider the following proposition.

Proposition 2. For given scalars $q, s, r$ and a matrix $\bar{H} \in \mathbb{R}^{N \times N}$ the following statements are equivalent

(i) The spectrum of $\bar{H}$ belongs to the algebraic stability domain $\sigma(\bar{H}) \subset \mathbb{D}(p)$ with

$$
\mathbb{D}(p)=\{z \in \mathbb{C} \mid p(\bar{z}, z)=r \bar{z} z-s(\bar{z}+z)+q<0\} .
$$

(ii) There exists a positive definite diagonal matrix $D$ and a orthogonal matrix $U^{T}=U^{-1}$ such that $H=$ $U^{T} \bar{H} U$ satisfies (10).

Proof. (i) $\rightarrow$ (ii) According to Lemma 2 condition (i) implies the existence of a positive definite solution of the inequality $-r \bar{H}^{T} X \bar{H}+s\left(X \bar{H}+\bar{H}^{T} X\right)-q X>0$. As $X=$ $X^{T}>0$ there exists a $U^{T}=U^{-1}$ orthogonal and $D>0$ diagonal such that $X=U D U^{T}$ (singular value decomposition of $X$ ). Left- and right-multiplying with $U^{T}$ and $U$ respectively does not change the inequality resulting in $-r U^{T} \bar{H}^{T} U D U^{T} \bar{H} U+s\left(D U^{T} \bar{H} U+U^{T} \bar{H}^{T} U D\right)-q D>0$ and with setting $H=U^{T} \bar{H} U$ in (10). For (ii) $\rightarrow$ (i) insert $H=U^{T} \bar{H} U$ into (10) and left-, right-multiply by $U, U^{T}$ which then states that for $\bar{H}$ (10) has the positive definite 
Table 1. Shape and size of algebraic domain $\mathbb{D}(p)$ for $H, \lambda_{i} \in \mathbb{C}$ represent the eigenvalues of $H$

\begin{tabular}{|c||c||c|}
\hline agent is & $(q, s, r)$ & $\mathbb{D}(p)$ \\
\hline \hline passive & $\left(0, \frac{1}{2}, 0\right)$ & open right halfplane \\
\hline IFP & $\left(0, \frac{1}{2}, r>0\right)$ & disc centered at $\frac{1}{2 r}$, radius $\frac{1}{2 r}$ \\
\hline sector-bounded $\left[k_{1}, k_{2}\right]$ & $\left(-1, \frac{1}{2}\left(k_{1}+k_{2}\right),-k_{1} k_{2}\right)$ & disc centered at $-\frac{k_{1}+k_{2}}{2 k_{1} k_{2}}$, radius $\frac{k_{1}-k_{2}}{2 k_{1} k_{2}}$ \\
\hline $\mathcal{L}_{2}$-stable & $\left(-1,0, \gamma^{2}\right)$ & disc centered at origin, radius $\gamma^{-1}$ \\
\hline
\end{tabular}

solution $X=U D U^{T}$. This is equivalent to the spectrum of $\bar{H}$ belonging to the algebraic stability domain according to Lemma 2.

Remark 5. Proposition 2 addresses the asymptotic stability case. In general, the Laplacian property (A.1) is not invariant under the transformation $T \in \mathcal{T}(D)$. This means that if assumption A1 is satisfied for $H$ it does not necessarily hold for $\tilde{H}$. In current research the extension to output-synchronization is investigated.

The algebraic stability domain $\mathbb{D}(p)$ (11) is given by a disc in the complex plane with the origin on the real axis with the center at $\frac{s}{r}$ and radius $\sqrt{\frac{s^{2}}{r^{2}}-\frac{q}{r}}$ for $r \neq 0$ as depicted in Fig. 2. For $r=0$, e.g. in the case of passive agents, the algebraic stability domain is given by a halfplane, see (11). Table 1 gives more examples for size and location of the algebraic stability domain for different $(q, s, r)$-dissipative agent characteristics. Note that a similar spectral interconnection characterization has been proposed in Hara et al. [2007] for agents with known and same linear time-invariant dynamics.

Remark 6. All the previous considerations apply generally for the undirected graph as well as for the directed graph. For the special case of an undirected graph, the spectrum of the interconnection matrix $H$ turns out to provide an appropriate characterization of the information topology to guarantee stability: If the interconnection matrix $H=H^{T}, H \in \mathcal{M}_{\sigma(H)}$ admits a solution of (10) for $D=\alpha I, \alpha>0$, then all symmetric matrices $H_{*}=H_{*}^{T}$, $H_{*} \in \mathcal{M}_{\sigma(H)}$ with the same spectrum also satisfy (10). This can be verified by replacing $H=U^{-1} H_{*} U$ with the orthogonal matrix $U^{-1}=U^{T}$ in (10), left-, rightmultiplying by $U, U^{T}$ and the fact that all symmetric matrices of the same spectrum are orthogonally similar. As a result inequality (10) rewrites in the same way with $H_{*}$ instead of $H$, i.e. is satisfied for every symmetric matrix $H_{*} \in \mathcal{M}_{\sigma(H)}$.

Important result of this section is the characterization of spectrum of the interconnection matrix $H$ as well as the finding, that in general the spectrum of the interconnection matrix is not sufficient to guarantee asymptotic stability/output-synchronization according to Theorem 1. The dependence on the coordinate system is a known issue for diagonal stabilizability in the sense of Corollary 1, see also Logofet [2005]. Here we also demonstrate that for the more general type Lyapunov inequalities (10). Note, however, that for any given spectrum out of the algebraic stability domain we can guarantee the existence of a interconnection structure and even more derive the class of co-spectral matrices that also satisfy the conditions of Theorem 1.

\section{APPLICATION TO OUTPUT-SYNCHRONIZATION PROBLEMS}

\subsection{Rendezvous - Position Coordination of Mobile Agents}

In the following example the position coordination of $N$ vehicles/robots with uncertain damping and inertia on a plane is considered. Each agent is a MIMO-system which satisfies the dynamics

$$
\begin{aligned}
M \ddot{z}+B \dot{z} & =u \\
y & =z
\end{aligned}
$$

where $z, \dot{z} \in \mathbb{R}^{2}$ is the position, velocity in the twodimensional Cartesian space, $u \in \mathbb{R}^{2}$. The matrices $M, B \in$ $\mathbb{R}^{2 \times 2}$ represent the uncertain positive definite inertia and damping matrix, respectively. They are assumed to be bounded by positive definite diagonal matrices $\bar{M}, \underline{B}$ such that $\bar{M} \geq M>0$ and $B \geq \underline{B}>0$. The damping matrix $B=B_{n}+K_{d}$ contains the natural damping of the agent and the local velocity feedback gain. Consider $V=\dot{z}^{T} M^{T} B^{-1} M \dot{z}+\dot{z}^{T} M z+\frac{1}{2} z^{T} B z$ as storage function. Each agent is $(Q, S, R)$-dissipative with $Q=0, S \frac{1}{2} I$, and $R=\underline{B}^{-1} \bar{M} \underline{B}^{-1}$ as obvious from computing $\dot{V} \leq u^{T} y+$ $u^{T} B^{-1} M B^{-1} u \leq u^{T} y+u^{T} \underline{B}^{-1} \bar{M} \underline{B}^{-1} u$. For simplicity we assume that $\bar{M}=\bar{m} I_{2}, \underline{B}=\underline{b} I_{2}$. The domain for the eigenvalues of the interconnection matrix $H$ in the complex plane is then given by a disc of radius $\frac{b^{2}}{2 \bar{m}}$ centered at $\frac{\underline{b}^{2}}{2 \bar{m}}$ on the real axis (11).

Example 2. For simulation we consider a network of 6 mass-damper agents (12) with a directed cyclic graph as information structure as shown in Fig. 3. Assuming that $\bar{m}=m$ and $\underline{b}=b$ the agents are dissipative with a common supply rate $(q, s, r)=\left(0, \frac{1}{2}, \frac{m}{b^{2}}\right)$. The local feedback gains and coupling gains are assumed to be all equal $\alpha_{i}=\beta_{i}>0$. We expect the system to be outputsynchronizing for $0<c \leq \frac{b^{2}}{2 m}$. The position trajectories for the parameters $m=1, b=1, c=\frac{1}{2}$ shown in Fig. 4(a) validate that. As discussed in earlier sections, the conditions presented in this paper have a sufficient character. They may come close, however, to necessity as the next simulation result shows. Consider therefore an overestimation of the damping parameter $b$ and let the true damping of the system be $20 \%$ smaller. As a result the system becomes unstable for the same interconnection matrix, see Fig.4(b).

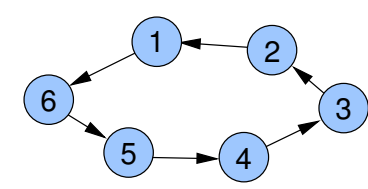

Fig. 3. Cyclic interconnection structure. 


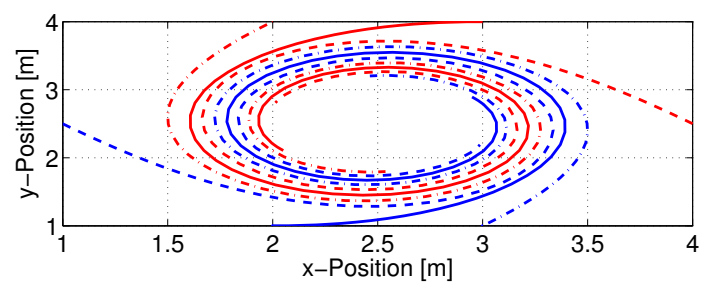

(a)

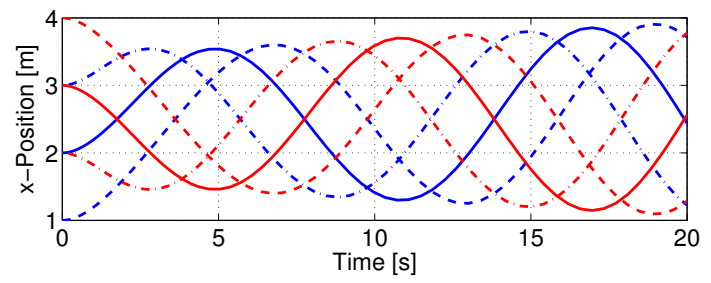

(b)

Fig. 4. Example of robot position coordination for agents with $m=1 \mathrm{~kg}, c=\frac{1}{2}$ : (a) output-synchronization conditions satisfied with $b=1 \mathrm{Ns} / \mathrm{m}$, and (b) conditions not satisfied with $b=0.8 \mathrm{Ns} / \mathrm{m}$

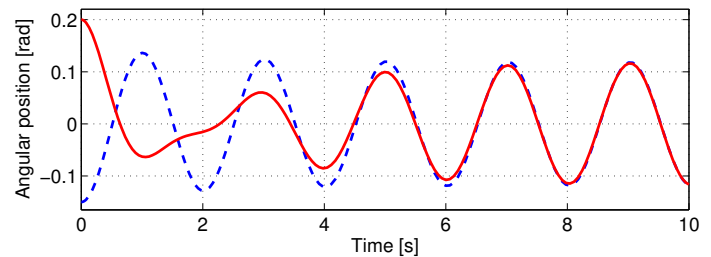

(a)

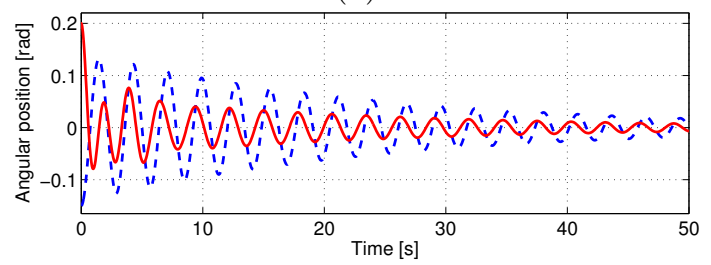

(b)

Fig. 5. Output-synchronization of two pendulums with (a) identical length $l_{1}=l_{2}=1 \mathrm{~m}$, synchronized oscillation; (b) different length $l_{1}=2 \mathrm{~m}, l_{2}=1 \mathrm{~m}$, all trajectories converge to zero.

\subsection{Synchronization of Coupled Pendulums}

We consider a similar example of coupled pendulums as in Chopra and Spong [2006], where we here also investigate the case for non-balanced graphs. Consider each pendulum having the length $l_{i}$ with the dynamics

$$
\ddot{q}_{i}+\frac{g}{l_{i}} \sin q_{i}=u_{i} ; \quad y_{i}=\dot{q}_{i}
$$

which is passive $\left(r=q=0, s=\frac{1}{2}\right)$ from $u_{i}$ to $\dot{q}_{i}$ with the positive definite storage function $V_{i}=\frac{1}{2} \dot{q}_{i}^{2}+\frac{g}{l_{i}}(1-$ $\cos q_{i}$ ). Considering two pendulums $N=2$ and choosing $H=\left[\begin{array}{cc}0.1 & -0.1 \\ -1 & 1\end{array}\right]$ assumption A1 is satisfied. According to Theorem 1 the system output-synchronizes and all trajectories converge to the largest invariant set where $\dot{q}_{1} \equiv \dot{q}_{2}$, i.e. where

$$
\frac{g}{l_{1}} \sin q_{1} \equiv \frac{g}{l_{2}} \sin q_{2}
$$

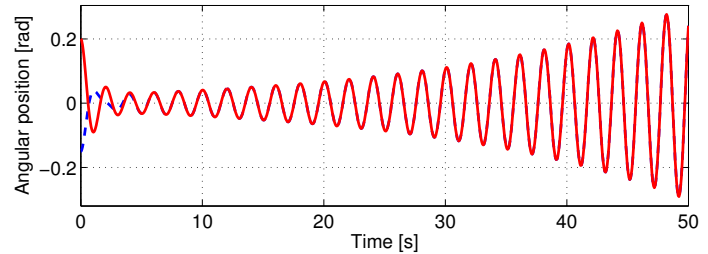

Fig. 6. Unstable behavior of two coupled identical pendulums with $l_{1}=l_{2}=1 \mathrm{~m}$; spectrum of $H$ does not satisfy the algebraic domain condition (11).

If the pendulums are identical $l_{1}=l_{2}$ then the largest invariant set is given by $q_{1} \equiv q_{2}$ and thus the pendulums synchronize as shown in Fig. 5(a). If the pendulums have different lengths the largest invariant set is given by $\dot{q}_{i}=$ $q_{i}=0$, i.e. the all trajectories eventually converge to zero as shown in Fig. 5(b). With the choice $H=\left[\begin{array}{cc}0.9 & -1 \\ -1 & 0.9\end{array}\right]$ the spectrum does not satisfy the algebraic domain condition of Proposition 2, (11) as one of the eigenvalues $\lambda_{1}=-0.1$, $\lambda_{2}=1.9$ has a negative real part. The coupled system is indeed unstable as shown in Fig. 6 .

From this example again we observe that the even though "only" sufficient conditions are presented the gap to necessity might be small.

\section{CONCLUSION}

In this paper we consider the stabilization and outputsynchronization of the class of dissipative agents with a quadratic supply rate. Main results concern the characterization and design of the information exchange structure. Here a linear protocol is assumed and as a result this structure can be represented by an interconnection matrix. Using a weighted Lyapunov approach outputsynchronization conditions are derived in terms of LMI's and using the equivalence to algebraic domains also in terms of spectral properties of the interconnection matrix. The design problem is adressed by an LMI. The results of this paper are successfully validated in simulations. Current work adresses the consideration of agents with dissipative uncertainties, the implementation of more sophisticated performance goals into the LMI synthesis and the time delay problem.

\section{ACKNOWLEDGEMENTS}

This work was supported in part by the Japanese Society for the Promotion of Science (JSPS). The first author is indebted to Professor Masayuki Fujita for his endless support. The constructive comments of the anonymous reviewers are highly appreciated

\section{REFERENCES}

M. Arcak. Passivity as design tool for group coordination. IEEE Transactions on Automatic Control, 52(8):13801390, August 2007.

S. Boyd. Convex Optimization of Graph Laplacian Eigenvalues. In Proceedings International Congress of Mathematicians, 2006.

N. Chopra and M. W. Spong. Passivity-based control of multi-agent systems. In From Everyday Physics 
to Human-Like Movements, pages 107-134. SpringerVerlag, Berlin, 2006.

J. A. Fax and R. M. Murray. Information Flow and Cooperative Control of Vehicle Formations. IEEE Transactions on Automatic Control, 49(9):1465-1476, 2004.

C. Godsil and G. Royle. Algebraic Graph Theory. Springer, New York, (US), 2000.

S. Hara, T. Hayakawa, and H. Sugata. Stability analysis of linear systems with generalized frequency variables and its applications to formation control. In Proceedings of the IEEE Conference on Decision and Control, 2007.

D. Hill and P. Moylan. The Stability of Nonlinear Dissipative Systems. IEEE Transactions on Automatic Control, 21(5):708-711, 1976.

D. Hinrichsen and A. J. Pritchard. Mathematical Systems Theory I. Springer, Berlin, 2005.

I.-A. F. Ihle, M. Arcak, and Fossen T. I. Passivity-based designs for synchronized path-following. Automatica, 43 (9):1508-1518, 2007.

D. Lee and M. W. Spong. Flocking of Multiple Inertial Agents on Balanced Graph. In Proceedings of the American Control Conference, pages 2136-2141, Minneaolis,US, 2006.

D. Logofet. Stronger-than-Lyapunov notions of matrix stability, or how "flowers" help solve problems in mathematical ecology. Linear Algebra and its Application, 398:75-100, 2005.

P. Moylan and D. Hill. Stability Criteria for Large-Scale Systems. IEEE Transactions on Automatic Control, 23 (2):143-149, 1978.

R. Olfati-Saber, J. A. Fax, and R. M. Murray. Consensus and Cooperation in Networked Multi-Agent Systems. Proceedings of the IEEE, 95(1):215-233, 2007.

D. Peaucelle, D. Arzelier, and R. Bertrand. Ellipsoidal sets for static output feedback. In Proceedings of the 15th IFAC Worldcongress, Barcelona (Spain), 2002.

W. Ren and R. W. Beard. Decentralized Scheme for Spacecraft Formation Flying via Virtual Structure Approach. AIAA Journal of Guidance, Control, and Dynamics, 27 (1):73-82, 2004.

R. Sepulchre, M. Jankovic, and P. Kokotovic. Constructive Nonlinear Control. Springer, New York, 1997.

J. C. Willems. Dissipative Dynamical Systems - Part I: General Theory. Arch. Rational Mechanics Analysis, 45:321-351, 1972a.

J. C. Willems. Dissipative Dynamical Systems - Part II: Linear Systems with Quadratic Supply Rates. Arch. Rational Mechanics Analysis, 45:352-393, 1972b.

\section{Appendix A. ALGEBRAIC GRAPH THEORY}

Directed/undirected graphs are commonly used to model the information exchange between the agents in a cooperative team, see Godsil and Royle [2000] for a comprehensive treatment of this matter.

A digraph (directed graph) consists of a pair $\mathcal{G}=(\mathcal{V}, \mathcal{E})$ where $\mathcal{V}=\{1,2, \cdots, N\}$ is a finite non-empty set of vertices and $\mathcal{E} \subseteq \mathcal{V} \times \mathcal{V}$ is the set of ordered pairs of distinct vertices, called edges. The pairs of vertices in an undirected graph are unordered. A directed path of length $l$ is a sequence of the form $v_{0}, \cdots, v_{l}$ of $l+1$ distinct vertices such that for every $i \in\{0, \cdots, l-1\},\left(v_{i}, v_{i+1}\right)$ is an edge.
A directed graph is strongly connected if any two vertices can be joined by a path. The adjacency matrix $\mathcal{A}=\left\{a_{i j}\right\}$ of a weighted digraph is defined as $a_{i i}=0$ and $a_{i j}>0$ if $(i, j) \in \mathcal{E}$ where $i \neq j$. The Laplacian matrix $\mathcal{L}=\left\{l_{i j}\right\}$ of a weighted digraph is defined as

$$
l_{i j}=\left\{\begin{array}{l}
-a_{i j} \text { if } i \neq j \\
\sum_{k} a_{i k} \text { otherwise } .
\end{array}\right.
$$

Note that there is only a connection from agent $j$ to agent $i$ if $l_{i j} \neq 0$.

The following propositions represent fundamental knowledge in algebraic graph theory but are taken over in the specific wording here from Fax and Murray [2004].

Proposition 3. Zero is an eigenvalue of $\mathcal{L}$, the associated vector eigenvector is $\mathbf{1}^{T}=[1, \cdots, 1]$.

Proposition 4. If $\mathcal{G}$ is strongly connected, the zero eigenvalue of $\mathcal{L}$ is simple.

\section{Appendix B. GENERALIZED LYAPUNOV EQUATION AND ALGEBRAIC STABILITY DOMAINS}

Linear matrix equations/inequalities may equivalently be expressed in terms of the a prescribed region of the complex plane where the eigenvalues of the matrix are lying in. Define therefore the domain $\mathbb{D}(p)$ in the complex plane characterized by the Hermitian polynomial $p: \mathbb{C} \times$ $\mathbb{C} \rightarrow \mathbb{C}$ as

$$
\mathbb{D}(p)=\{z \in \mathbb{C}: p(\bar{z}, z)<0\},
$$

where

$$
p\left(z_{1}, z_{2}\right)=\sum_{i=1}^{l} \sum_{j=1}^{l} c_{i j} z_{1}^{i-1} z_{2}^{j-1}=l^{T}\left(z_{1}\right) C l\left(z_{2}\right),
$$

with $C=\left\{c_{i j}\right\} \in \mathcal{H}_{l}, i, j \in\{1, \cdots, l\}, l(z)=$ $\left[1, z, z^{2}, \cdots, z^{l-1}\right]^{T}$. For a given matrix $A \in \mathbb{C}^{n \times n}$ and $Y \in \mathcal{H}_{n}$ the matrix equation

$$
\sum_{i=1}^{l} \sum_{j=1}^{l} c_{i j}\left(A^{*}\right)^{i-1} X A^{j-1}=-Y,
$$

is called the generalized Lyapunov equation with respect to $p(\cdot, \cdot)$ where $X \in \mathcal{H}_{n}$. The following lemma provides a connection between the generalized Lyapunov equation (B.3) and the domain $\mathbb{D}(p)$ (B.1).

Lemma 2. (Hinrichsen and Pritchard [2005]). Consider the matrix $A \in \mathbb{C}^{n \times n}$ and the domain $\mathbb{D}(p)$ given by (B.1). Then the following statements are equivalent:

(i) $\sigma(A) \subset \mathbb{D}(p)$

(ii) There exists a positive definite matrx $Y \in \mathcal{H}_{n}$ such that the generalized Lyapunov equation (B.3) yields a unique positive definite solution $X \in \mathcal{H}_{n}$.

If in addition, $\sigma(C)$ contains a single negative eigenvalue then the following statement is equivalent to (i) and (ii) above:

(iii) For any $Y \in \mathcal{H}_{n}$, the generalized Lyapunov equation (B.3) yields a unique positive definite solution $X \in \mathcal{H}_{n}$.

In consequence, the problem of finding the spectral location of a matrix can be re-cast as an LMI problem and vice versa. 